\title{
COVID-19 and its impact on CCFP(EM) residency training
}

\author{
Avik Nath $^{1}\left[\right.$ - Cheryl Hunchak ${ }^{2} \cdot$ Sheila Smith $^{3} \cdot$ Jason Fedwick ${ }^{4} \cdot$ Erich Hanel $^{5} \cdot$ Peter Rogers $^{6}$ \\ Received: 29 January 2021 / Accepted: 11 May 2021 / Published online: 22 May 2021 \\ (c) The Author(s), under exclusive licence to Canadian Association of Emergency Physicians (CAEP)/ Association Canadienne de Médecine d'Urgence \\ (ACMU) 2021
}

Keywords CCFP(EM) $\cdot$ Enhanced skills $\cdot$ Emergency medicine

\section{Introduction}

The COVID-19 pandemic has significantly impacted Canadian residency training programs [1-7]. In particular, the impact on enhanced skills training programs in emergency medicine, CCFP(EM), have been broad, impacting clinical rotations, academic programming, resident recruitment, the CaRMS (Canadian Resident Matching Service) process, certification examinations, and importantly, resident wellness. The compact nature of a 1-year training program, and significant variability of training at each site has led to unique educational challenges nationally.

\section{Impact on clinical rotations and academic programming}

The CCFP(EM) program is fast-paced and residents are exposed to a high volume of acute and critical patient care during their 1-year of training. In March 2020, the first wave

\section{Avik Nath}

anath@toh.ca

1 Department of Emergency Medicine, The Ottawa Hospital, Civic Campus, University of Ottawa, 1053 Carling Ave., Ottawa, ON K1Y 4E9, Canada

2 Department of Family and Community Medicine, University of Toronto, Toronto, ON, Canada

3 Department of Academic Family Medicine, University of Saskatchewan, Regina, SK, Canada

4 Department of Emergency Medicine, University of Calgary, Calgary, AB, Canada

5 Division of Emergency Medicine, Department of Family Medicine, McMaster University, Hamilton, ON, Canada

6 Discipline of Emergency Medicine, Faculty of Medicine, Memorial University, St. John's, NL, Canada of the COVID-19 pandemic changed this, precipitating an unprecedented drop in emergency department (ED) volumes across the country [8]. Exposure to cardinal ED presentations diminished during 'lockdown' in affected provinces. Accordingly, EM residents in the 2019-2020 cohort likely saw lower caseloads from March to June.

Relative to many other residency training programs, emergency medicine (EM) residents were spared disruptions in their core rotations and were able to continue providing in-person clinical care. However, off-service rotations were often cancelled or cases substantially reduced. Resuscitation of critically ill ED patients were now run as "Protected Code Blues" (PCBs), either by intubation teams comprised of anaesthetists, or exclusively by staff ED physicians. This practice led to decreased, or at least variable, opportunities for EM residents to lead resuscitations and manage airways, as well as to perform other aerosol generating medical procedures.

Fourteen-day self-isolation policies in some provinces, could represent half of a residents' rotation, and, the time required to make up is challenging in a 1-year residency. Innovative approaches to this included having asymptomatic residents 'front load' their ED shifts into the first 2 weeks of the rotation, followed by 2 weeks of mandatory self-isolation before starting a next rotation, or arranging alternate rotations on short notice in departments without declared outbreaks.

The effect on academic programming in Canadian emergency medicine residencies and strategies to address these concerns have been published elsewhere [1,2]. Varying strategies outlined [1] were implemented with consideration of the unique 1-year training of CCFP(EM) programs. As for all training programs, academic teaching sessions had to rapidly adjust to virtual platforms. Furthermore, residents had completed the majority of their clinical and academic training when the pandemic was declared, but still had critical consolidation tasks to complete, including 
advanced ultrasound training, examination review, and transition to practice type academic sessions. These critical learning opportunities in particular need timely implementation of novel strategies considering the short nature of the training program.

The pandemic has highlighted the lack of flexibility inherent in a 1-year training program. Potential strategies to mitigate this include increasing the use of simulation and consideration of integrated 3-year FM/EM training programs. Increased use of simulation could ensure a minimum acceptable exposure to critically ill patients in the event that authentic exposures do not occur. An integrated 3-year program, such as at Dalhousie University, could provide the necessary flexibility around re-scheduling rotations, as well as critical consolidation of tasks during future pandemics.

\section{Resident recruitment and CARMS}

Entry into CCFP(EM) residency programs is competitive and informed by the Family Medicine (FM)/Enhanced Skills CaRMS match. Given its September application deadline, most FM residents considering EM training would have planned summer EM electives as a key component of their application strategy. However, in response to the pandemic, many postgraduate departments placed a moratorium on visiting elective residents. In addition, several jurisdictions instituted 2-week self-isolation periods for anyone visiting from outside the province. Residents pursue electives at sites of interest to assess whether a program is the right fit for them, and to get reference letters from preceptors at that site. Programs rely on reference letters from elective sites as another data point in a candidate's application. This has led to a significant adjustment in how programs select interview candidates.

The pandemic likely had little effect on residency programs' candidate file review process nationally, as applications are available for faculty review via the CaRMS online portal. The same cannot be said for the interview process. Traditionally residency interviews are held in-person, barring exceptional circumstances. This has been viewed as an opportunity for candidates to 'showcase themselves' and for programs to display their city, and life outside of residency training. In 2020, all residency selection interviews were carried out virtually. In particular, smaller programs rely on in-person interviews to showcase the culture of their city and the advantages of their smaller department when candidates visit. While virtual interviews have some perceived limitations and inequities, they do offer several advantages including substantial cost savings, a reduced carbon footprint, and limiting time missed from rotations. Whether virtual interviews are a better way forward for the residency application process is yet to be determined.

\section{Certification exams}

The COVID-19 pandemic led to significant disruptions in the ability of the College of Family Physicians of Canada (CFPC) to safely administer its certification exam. The CFPC certification exam was postponed from Spring 2020 to Fall 2020 at which time residents were just starting their residency year. This led to a re-organization of program curricula and time off a residents' clinical rotation to accommodate resident preparation for the exam. Many residents reported increased stress from feeling pulled in two directions: one, studying for their CPFC certification exam; and the other, studying their Enhanced Skills EM material.

The September 2020 CFPC Examination of Added Competence in Emergency Medicine, consisted only of the written portion. The oral component of the exam was cancelled. This led to a record number of practice eligible physicians challenging the CFPC(EM) exam.

Holding only the written portion of the certification exam adversely affected candidates from 2019 who had a failed standing in the exam. Candidates who were not successful in the oral component of the 2019 exam were not eligible to write the exam in 2020 . They will have to wait at least 2 years to challenge the exam again, should the oral component of the exam be reinstated in 2021. Candidates who were unsuccessful in the written portion in 2019 initially were not allowed to challenge the CCFP(EM) exam in 2020. After much deliberation, the CFPC allowed those candidates to write the exam, but informed eligible candidates with only 1-month to prepare. For the 2021 exam, the CFPC has indicated they are hoping to administer both the oral and written components of the exam; however, this will only be confirmed in Spring 2021 with consideration of provincial and federal guidelines.

\section{Resident wellness}

Canadian EM residents are on the frontline of the battle against COVID-19. Levels of burnout rise with each year of training and exposure to the stresses of providing emergency care [9]. COVID-19 is making things worse. Residents providing care to COVID-19 patients report higher levels of burnout [9]. Residents have faced pandemic-related disruptions to their academic curriculum, clinical rotations, their certification exam and isolation in all spheres of their life: they face personal risk of infection and the chance of bringing the disease home to families, 


\section{Covid-19 and CCFP(EM) \\ Programs: Lessons learned}

Avik Nath, MD, MSc

Cheryl Hunchak, MD, MSc

Sheila Smith, MD

Jason Fedwick, MD, PhD

Erich Hanel, MB, MSc

Peter Rogers, MD

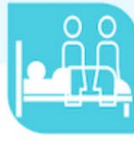

\section{Simulation}

Simulation should be used to cover key resuscitation scenarios that are not guaranteed to be experienced on clinical rotations with higher infection control standards and lower patient volumes.

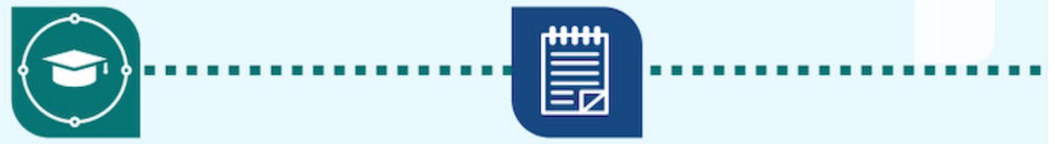

Academic Curriculum

Academic programming must be easily accessible virtually and implemented without delay due to the nature of a 1-year training program

\section{Recruitment}

Programs need to adapt to advertising their program, providing opportunities for potential candidates to meet residents and faculty without travel, and having a platform to reliably do virtual interviews

\section{"." \\ Virtual Oral Exams}

Reliable, virtual oral exam platforms need to be sought out with clear communication and planning from licensing bodies to ensure timely, welladvertised, and well-thought-out criteria for exam eligibility

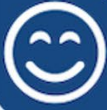

\section{Resident Wellness}

Strategies for resident wellness need to be implemented early to help with resident resiliency during an already difficult year

Infographic by Dr. Hans Rosenberg

Fig. 1 Covid-19 and CCFP(EM) Programs: Lesson learned 
friends and neighbors; time with loved ones, vacations, social, and team building events are all on hold; and human connection has been replaced by virtual meetings.

EM residency programs need to continue focusing on resident wellness. After the pandemic, we cannot be allowed to return to a "normal" where $80 \%$ of attendings report symptoms of burnout $[10,11]$. In less than a year, new treatments and vaccines for COVID-19 were developed. As a specialty we need to advocate for continued study of burnout to better support our trainees as they enter clinical practice. Real system-level solutions have been proposed [12]. Evidence already exists that well physicians are ultimately more efficient, cost the system less, and provide better patient care [13]. Emergency medicine, with the highest levels of burnout, could lead the charge in finding the cure.

\section{Conclusion}

The COVID-19 pandemic has significantly impacted CCFP(EM) programs across the country. Programs have been meeting these challenges and the valuable lessons learned from this pandemic (Fig. 1) will help residency programs deal with future public health emergencies.

Acknowledgements The authors would like to extend their gratitude to Dr. H. Rosenberg for creation of the infographic, and all CCFP(EM) National Emergency Medicine Directors for their contributions to this article.

Author contributions All authors contributed to the writing of the manuscript.

Funding None.

\section{Declarations}

Conflict of interest The authors declare that they have no conflict of interest.

\section{References}

1. Mok G, Schouela N, Thurgur L, Ho M, Hall AK, Caudle J, et al. Resident learning during a pandemic: Recommendations for training programs. CJEM. 2020;22(5):617-21.
2. Thoma B, Woods R, Patocka C. Context: How COVID-19 exposed key factors of emergency medicine education. CJEM. 2020;22(5):561-2.

3. Wang DC, Emmad Q, Jiayi TM, Eric B. COVID-19 pandemic on Canadian radiology residency education-impact and solutions: University of Toronto perspective. Can Assoc Radiol J. 2020. https://doi.org/10.1177/0846537120940299.

4. Odedra D, Chahal BS, Patlas MN. Impact of COVID-19 on Canadian radiology residency training programs. Can Assoc Radiol J. 2020;71(4):482-9. https://doi.org/10.1177/0846537120933215 (Epub 2020 Jun 11. PMID: 32522010; PMCID: PMC7290107).

5. O'Brien J, Deck M, Goncin U, Chaya M. Impact of the COVID-19 pandemic on anesthesia residency education: Les effets de la pandémie de la COVID-19 sur la formation des résidents en anesthésie. Can Med Educ J. 2020;11(5):e126-e128. https://journalhos ting.ucalgary.ca/index.php/cmej/article/view/70457. Accessed 12 Jan 2021.

6. Chan EP, Stringer L, Wang PZ, Dave S, Campbell JD. The impact of COVID-19 on Canadian urology residents. CUAJ. 2020;14(6):E233-E236. https://cuaj.ca/index.php/journal/artic le/view/6713. Accessed 12 Jan 2021.

7. Mann U, Nayak JG. The potential impact of COVID-19 on the Canadian Resident Matching Service: unique future challenges faced by urology residency programs and applicants. Can Urol Assoc J. 2020;14(5):E167-8. https://doi.org/10.5489/cuaj.6616.

8. https://www.cihi.ca/en/covid-19-resources/impact-of-covid-19on-canadas-health-care-systems/how-covid-19-affected. Accessed 9 Dec 2020

9. Kannampallil TG, Goss CW, Evanoff BA, Strickland JR, McAlister RP, Duncan J. Exposure to COVID-19 patients increases physician trainee stress and burnout. PLoS ONE. 2020;15(8):e0237301.

10. Stehman CR, Testo Z, Gershaw RS, Kellogg AR. Burnout, drop out, suicide: physician loss in emergency medicine, Part I. West J Emerg Med. 2019;20(3):485-94.

11. Lim R, Aarsen KV, Gray S, Rang L, Fitzpatrick J, Fischer L. Emergency medicine physician burnout and wellness in Canada before COVID19: a national survey. CJEM. 2020;22(5):603-7.

12. Shanafelt TD, Dyrbye LN, West CP. Addressing physician burnout: the way forward. JAMA. 2017;317(9):901-2.

13. Mateen FJ, Dorji C. Health-care worker burnout and the mental health imperative. Lancet. 2009;374(9690):595-7. 